Research Article

\title{
The Influence Mechanism and Optimization of the Sensor Network on the MS/AE Source Location
}

\author{
Nan Li $\mathbb{D}^{1,2}$ Maochen Ge, ${ }^{3}$ Enyuan Wang, ${ }^{1,4}$ and Shaohua Zhang ${ }^{1}$ \\ ${ }^{1}$ State Key Laboratory of Coal Resources and Safe Mining, China University of Mining and Technology, Xuzhou, \\ Jiangsu 221116, China \\ ${ }^{2}$ School of Mines, China University of Mining and Technology, Xuzhou, Jiangsu 221116, China \\ ${ }^{3}$ Mining Engineering and Nuclear Engineering Department, Missouri University of Science and Technology, Rolla 65409, \\ MO, USA \\ ${ }^{4}$ School of Safety Engineering, China University of Mining and Technology, Xuzhou, Jiangsu 221116, China
}

Correspondence should be addressed to Nan Li; cumtlinan@126.com

Received 31 December 2019; Revised 24 July 2020; Accepted 19 August 2020; Published 7 September 2020

Academic Editor: Giuseppe Petrone

Copyright (C) $2020 \mathrm{Nan} \mathrm{Li}$ et al. This is an open access article distributed under the Creative Commons Attribution License, which permits unrestricted use, distribution, and reproduction in any medium, provided the original work is properly cited.

\begin{abstract}
The sensor network layout is a key factor affecting the accuracy and stability of the microseismic/acoustic source (MS/AE) location. Based on the arrival-time-difference principle, the hyperbolic/hyperboloidal governing equations for the source location are derived. The nonuniformity geometrical characteristics of hyperbolic/hyperboloidal field for the source location are obtained. The sensor network does not induce any location errors; it only affects the source location accuracy by amplifying the existing errors in the input data during the source location process. Also, this amplication effect of the input data errors is characterized by nonuniformity because of the nonuniformity of the hyperbolic/hyperboloidal field. Furthermore, two basic effects, the geometrical spreading and the directional control, of the sensor network are investigated, and the three-dimensional space quantitative models of these two effects are established, respectively. The influence of the wave velocity error and arrival time error on the source location accuracy is analytically compared, and the propagation characteristics of these two types of errors during the source location process are revealed. The concepts of critical arrival-time difference and critical hyperbola/hyperboloid are proposed. Based on these two concepts, the monitoring area can be divided into two regions where the source location accuracy is controlled by the velocity error and the arrival time error, respectively. The concept of direction angle of paired sensors is proposed, and the relationship between the source location and the layout of four typical paired sensors is studied. Finally, the principles of sensor network optimization are determined.
\end{abstract}

\section{Introduction}

It is generally accepted today that rock emits seismic signals when it is stressed and/or deformed. The microseismic (MS)/ acoustic emission (AE) technique refers to a geophysical monitoring technology that studies the internal structure and stability, fracture mechanism, and morphology of rock mass by using the seismic signals. In the recent years, the MS/AE technique has been widely used in monitoring and early warning of coal and rock dynamic disasters, hydraulic fracture (HF) monitoring, and stability monitoring of underground structures such as mines, tunnels, and natural gas, and petroleum storage caverns, as well as surface structures such as dams, slopes, and foundations [1-3]. A high accuracy source location is the first step in studies of the MS/AE technique. The factors that influence the source location accuracy may be divided into two types: input data errors and systematic bias $[4,5]$. The source location accuracy is definitely affected if there are errors in the input data such as the velocity model and the arrival time. The influence of the systematic error is mainly caused by the sensor network layout. Some effects of the sensor network relative to location accuracy have been noted; for example, source location accuracy inside a sensor network is usually better than that outside this network. But, the general influence of the sensor network is still not clear. 
Because the errors associated with the input data are unavoidable, the realistic solution is to reduce the impact of these input errors. So, many advanced source location algorithms and optimization analysis methods are developed, such as Geiger's method, genetic algorithms, the simplex method, the double-difference method, and the particle swarm optimization method [6-10]. On the other hand, the stability and accuracy of the source location are also dependent on the sensor network layout. The sensor network geometry and its mechanism in the control of source location are critical problems needing study in detail $[3,11]$. Some people studied the source location efficiency for particular sensor network geometries, such as triangle, quadripartite, and hexagon [12-14]. Kijko proposed a general method to the distribution of seismic sensors based on the D-optimality theory. Also, he developed a procedure for obtaining the optimal spatial distribution of MS sensors in mines by using D-optimality theory [15-17]. The D-optimality method and its variants were widely applied in natural and induced earthquakes [18-22]. Based on the D-optimization theory, Gong et al. [4, 23] proposed a principle to choose the optimal channel numbers and created a model of the expected epicenter and hypocenter error by using the numerical emulation experiment. Finally, they built an optimal design and assessment system of the MS network. Mendecki developed a method for optimal design of mine seismic networks based on the C-optimality theory [24]. The research results of Jia and $\mathrm{Li}$ indicated that the source location accuracy depends upon the geometry between the sources and MS sensors when the wave velocity model is determined [25]. An optimization of the MS network was carried out in the Dongguashan Copper Mine by Tang et al. [21], and they designed some different schemes of the MS network. The optimum MS network is obtained by calculating the errors of source location and system sensitivities associated with these different networks. Wang et al. studied the relationship between the MS station coordinates deviation and source location accuracy, and results show that the source location errors increase with errors of the MS sensor station [26]. Jia et al. found that the impact of the sensor density on the source location accuracy can be divided into two parts: the number of sensors and sensor plane quantity [27]. Stabile et al. developed a method for evaluating network performance in surface and borehole seismic monitoring. This method can estimate the location and origin time errors of seismic events with different magnitudes by a specified network geometry [28]. According to Stabile's method, Landro et al. evaluated the performance of sensor networks with varying the station density and geometry in the induced MS monitoring. The results show that the station density and the noise level represent the crucial parameters for the seismic network performances [29]. LI et al. studied the influence law of the MS/AE source location affected by the sensor network, velocity model, and arrival time by laboratory tests $[5,11]$. They also studied two types of multiple solutions related to the sensor network for the source location if the arrival-time-difference principle is used [30].

In summary, previous studies mainly focused on the optimization and evaluation of the specific sensor network. For example, the performance of a network is usually evaluated after installation by plotting the contour map of the location error in the monitoring area. However, compared to the importance of sensor network geometry, it is remarkable how little attention has been paid to the effect of the sensor network on the MS/AE source location. Therefore, based on the space geometry theory and arrival-timedifference principle, we first demonstrated that why and how the sensor network has such an important influence on the MS/AE source location. Then, propagation characteristics of input data errors, such as wave velocity error and arrival time error, are determined. Finally, the optimization principle of the sensor network layout is proposed.

\section{Hyperbolic/Hyperboloidal Governing Equation of the Source Location and Its Geometrical Distribution Characteristics}

2.1. The Hyperbolic/Hyperboloidal Governing Equation. Suppose $\mathrm{S}\left(x_{0}, y_{0}, z_{0}, t_{0}\right)$ and $\mathrm{T}_{i}\left(x_{i}, y_{i}, z_{i}, t_{i}\right)$ are the MS or $\mathrm{AE}$ source and the $i$ th sensor, respectively. It is very difficult to identify $S$ waves because the MS and AE monitoring area is small. So, $P$-wave velocity is the most commonly used velocity in the MS and AE source location. Also, the source location equation based on the arrival-time-difference principle can be expressed as

$$
\sqrt{\left(x_{i}-x_{0}\right)^{2}+\left(y_{i}-y_{0}\right)^{2}+\left(z_{i}-z_{0}\right)^{2}}=v_{P}\left(t_{i}-t_{0}\right)
$$

where $x_{0}, y_{0}, z_{0}$ and $x_{i}, y_{i}, z_{i}$ are the coordinates of the source and the $i$ th sensor, respectively. $t_{0}$ and $t_{i}$ are the origin time of the source and the observed arrival time of the $P$ wave at the $i$ th sensor. $v_{P}$ is the $P$-wave velocity. Also, $i=1,2, \ldots, N$, and here, $N$ denotes the number of this equation.

Let the observed arrival time of any two sensors, $T_{i}$ and $T_{j}$, be $t_{i}$ and $t_{j}$, and $t_{i}<t_{j}$. Subtracting the $i$ th equation from the $j$ th equation, which are all defined by system of equation (1), gives the source location-governing equation [30]:

$$
\sqrt{\left(x_{j}-x_{0}\right)^{2}+\left(y_{j}-y_{0}\right)^{2}+\left(z_{j}-z_{0}\right)^{2}}-\sqrt{\left(x_{i}-x_{0}\right)^{2}+\left(y_{i}-y_{0}\right)^{2}+\left(z_{i}-z_{0}\right)^{2}}=v\left(t_{j}-t_{i}\right) \text {. }
$$

According to the geometry theory, equation (2) represents a hyperboloid with the sensors $T_{i}$ and $T_{j}$ as the focal points, which indicates that the path of the source locations determined by two sensors is a hyperboloid in space. For a 
two-dimensional source location, the source location-governing equation (2) reduces to

$$
\sqrt{\left(x_{j}-x_{0}\right)^{2}+\left(y_{j}-y_{0}\right)^{2}}-\sqrt{\left(x_{i}-x_{0}\right)^{2}+\left(y_{i}-y_{0}\right)^{2}}=v_{p}\left(t_{j}-t_{i}\right) \text {. }
$$

Equation (3) represents a hyperbola, with sensor $T_{i}$ and $T_{j}$ as the foci, in a plane. According to analytical geometry, the difference of distances of any point on this hyperbola from its foci is a constant, say $2 a_{i j}$, which satisfies $2 a_{i j}=v_{p}\left(t_{j}-t_{i}\right)$. Therefore, the path of the potential source determined by two sensors in the plane is a hyperbola. In other words, any point on this hyperbola may be the potential source. So, the geometrical meaning of the arrivaltime-difference source location method is looking for the intersection point of different hyperbolas. As shown in Figure 1, two hyperbolas generated by three sensors in a plane and the intersection point of hyperbola I and II is the source.

2.2. Nonuniformity Geometrical Characteristics of the Hyperbolic Field of the Source Location. According to equation (3), the distance difference of any point on the hyperbola focusing on the sensors $T_{i}$ and $T_{j}$ from its foci is a constant which is equal to $2 a_{i j}$, and $2 a_{i j}=v\left|t_{j}-t_{i}\right|$. Thus, the position and the shape of a hyperbola just depend on the value of $a_{i j}$ when the positions of the sensors (foci) are determined. Based on the geometry of a hyperbola and the arrival-timedifference source location principle, $2 a_{i j}$ satisfies the following relationship:

$$
0 \leq 2 a_{i j} \leq 2 c_{i j},
$$

where $2 c_{i j}$ is the distance between the foci.

As shown in Figure 2, when the value of $2 a_{i j}$ is equal to zero, the source locates on the perpendicular bisector (denotes as line $L$ ) of the line jointing the two sensors. When $2 a_{i j}$ is $2 c_{i j}$, the source is on a focus or on the outside of the line jointing the foci. Also, a hyperbola field is formed (Figure 2) when the value of $2 a_{i j}$ increases from 0 to $2 c_{i j}$. In general, the smaller the value of $2 a_{i j}$, the closer the hyperbola to the line $L$. Besides, the density and the directions of hyperbola in this field are different.

Based on the source location-governing equation, the source is always located somewhere on one of the hyperbolas whose value of the real axis is equal to $2 a_{i j}=v\left|t_{j}-t_{i}\right|$. It means that the shape and position of a hyperbola that contains the source is affected by the parameters of arrival time and wave velocity. However, in practice, $2 a_{i j}$ always deviates from its true value because there are always some errors in arrival time and wave velocity. If the input data error is assumed equal to $\pm \delta a$, the calculating source will jump to a place on the adjacent hyperbola which controlled by the value of $\left(2 a_{i j} \pm \delta a\right)$.

As shown in Figure 2, there are five different sources in the hyperbolic field, and these five sources will incorrectly locate on the neighbouring hyperbolas if there are errors in the input data. For the same input error, say $\delta a=0.2 c$, it clearly shows that the location errors are different of these five sources. Generally speaking, the error increases as the sources are located further from the origin point $O$. In order to get good location accuracy, the real source need be at a point where the hyperbola corresponding to $\left(2 a_{i j}\right)$ and $\left(2 a_{i j} \pm \delta a\right)$ are close enough. As a result, the location error caused by the "source jumping effect" associated with the sensor network can be controlled. Otherwise, the location error will be amplified. Therefore, the higher the density of hyperbolic field in the vicinity of the source is, the better the source location accuracy there will be. Unfortunately, the hyperbolic field associated with sensor network is nonuniform, and the density of hyperbolas varies from point to point in the hyperbolic field. It makes the source location accuracy heavily dependent on the relative position of the true source and the sensor network layout.

From the abovementioned analysis, some important conclusions associated with sensor network layout and hyperbolic field are summarized as follows: the sensor network layout itself does not cause any location errors; it only amplifies input data errors already existed. The essence of this amplification in terms of hyperbolic geometry is that the calculated source is incorrectly located on an adjacent hyperbola rather than on the one associated with the true source. In addition, the effect of this amplification is not uniform, and this nonuniform amplification effect is due to the nonuniformity of the hyperbolic field. The results indicate that the location error is highly dependent on the position of the true source relative to the sensor network layout.

\section{The Mechanism of the Sensor Network in the Control of the Source Location}

3.1. Geometrical Spreading Effect of the Sensor Network. Since the source location accuracy in the monitoring area is due to the nonuniformity of the associated hyperbolic field, as seen in Figure 2, the line segment between the two sensors (or the foci) can be regarded as the original line where all hyperbolas are evenly spaced. But, the interval between the adjacent hyperbolas becomes larger and larger along these hyperbolas spread into the space. This effect associated with the nonuniform hyperbolic field is defined as the geometrical spreading effect. The density of hyperbolas in the quickest change direction is used to study the geometrical spreading effect quantitatively. Based on the geometry and mathematics theory, it is known that hyperbolas and ellipses are orthogonal when they are confocal. So, the quickest change direction of the hyperbola is the direction along its confocal ellipse.

Based on the space geometry theory, the geometrical spreading quantitative formulas can be derived as follows.

As shown in Figure 3, sensors $T_{1}$ and $T_{2}$ are the foci of the hyperbola and ellipse. $A_{1} A_{2}$ is the real axis of the hyperbola with the length of $2 a$, and $B_{1} B_{2}$ is the imaginary axis which has the length of $2 b . E_{1} E_{2}$ is the long axis of the ellipse which has the length of $2 e$, and $F_{1} F_{2}$ is the minor axis with the length of $2 f$. The hyperbola and ellipse in this confocal system can be expressed by the following equations: 


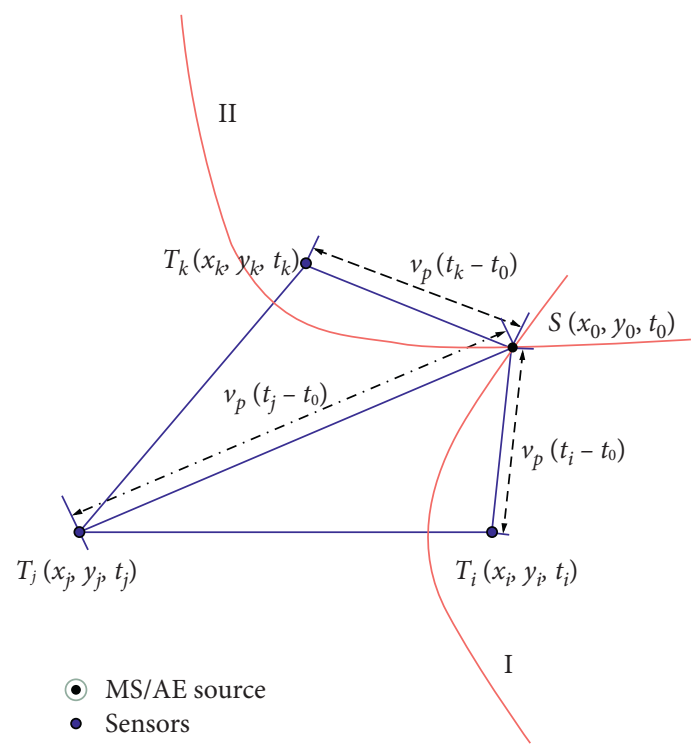

Figure 1: MS/AE source determined by two hyperbolas on a plane.

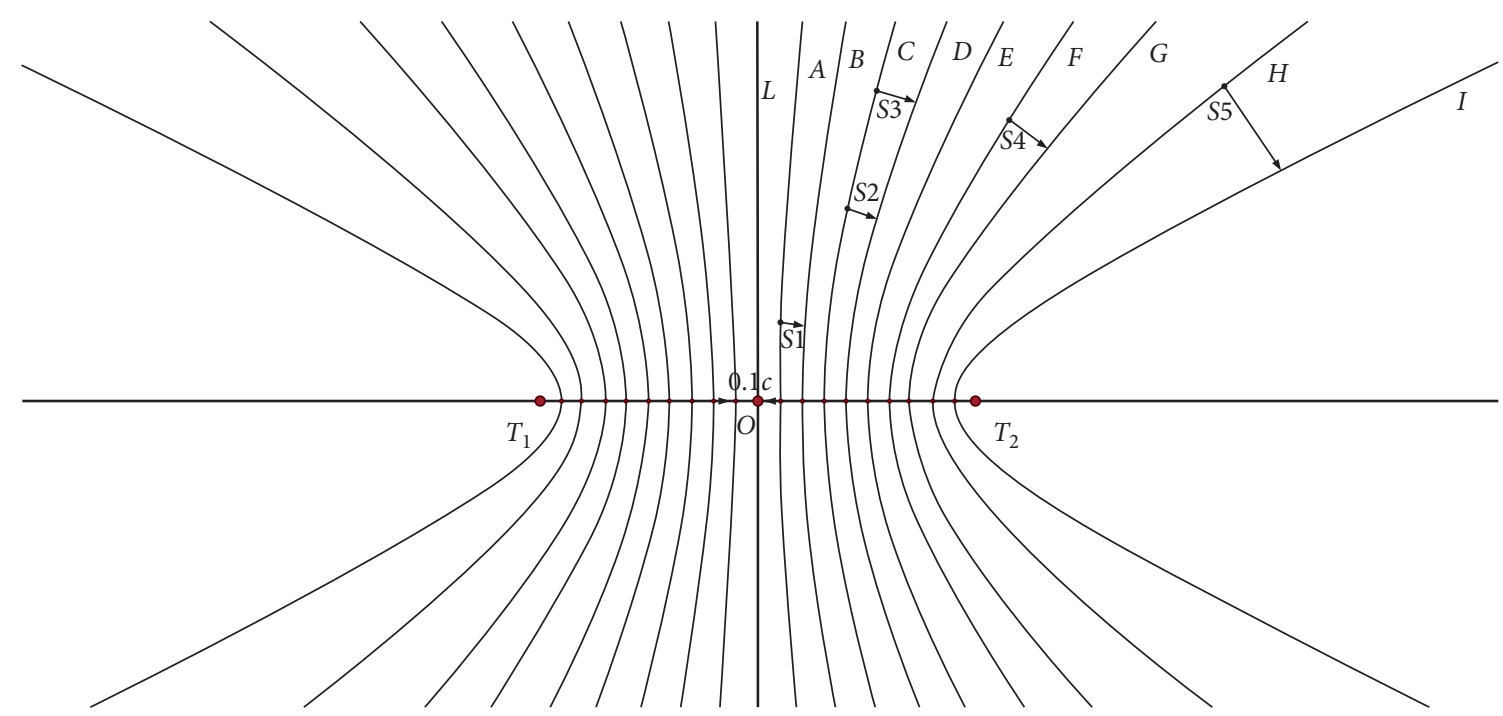

FIgURE 2: Hyperbolic field associated with two MS sensors $T_{i}$ and $T_{j}$.

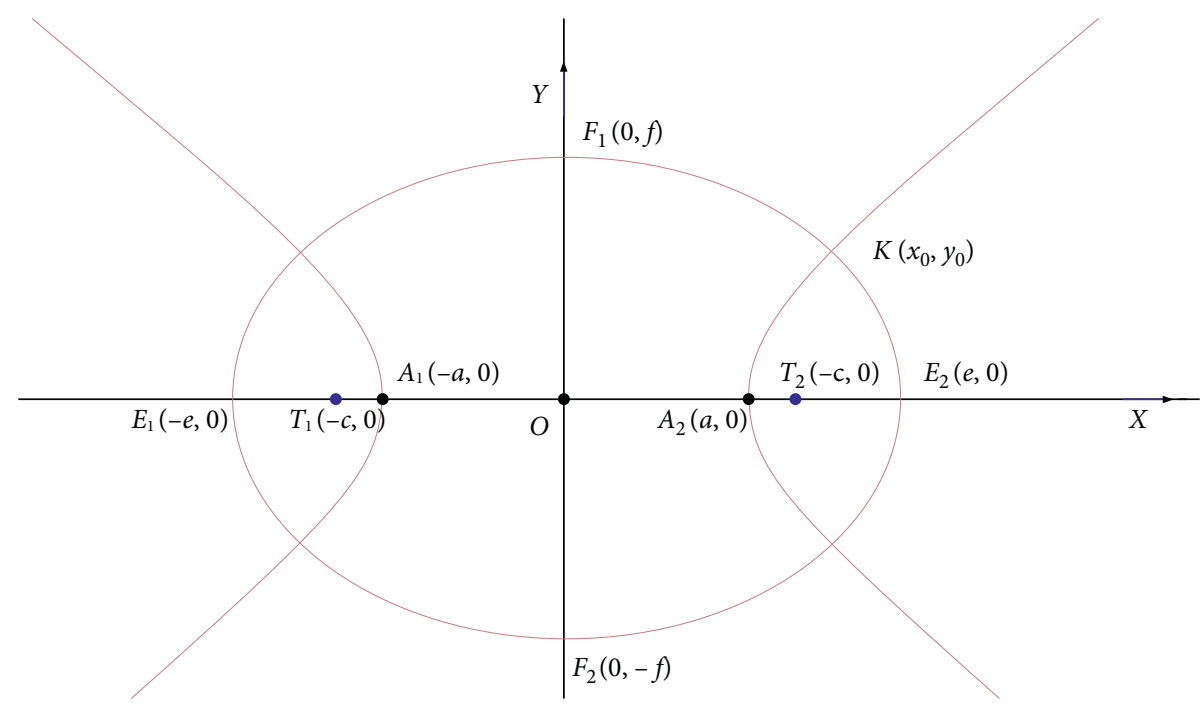

FIgURE 3: The confocal ellipse and hyperbola with foci $T_{1}$ and $T_{2}$. 


$$
\begin{aligned}
& \frac{x^{2}}{a^{2}}-\frac{y^{2}}{b^{2}}=1, \\
& a^{2}+b^{2}=c^{2}, \\
& \frac{x^{2}}{e^{2}}+\frac{y^{2}}{f^{2}}=1, \\
& c^{2}+f^{2}=e^{2} .
\end{aligned}
$$

As shown in Figure 4, hyperboloid of two sheets and ellipsoid are generated by the rotation, about the $X$-axis, of corresponding hyperbola and ellipse located in the XY plane. The equations of the hyperboloid and ellipsoid are as follows:

$$
\begin{aligned}
& \frac{x^{2}}{a^{2}}-\frac{y^{2}+z^{2}}{b^{2}}=1, \\
& \frac{x^{2}}{e^{2}}+\frac{y^{2}+z^{2}}{f^{2}}=1 .
\end{aligned}
$$

The trajectory of the intersecting points of the confocal hyperboloid and ellipsoid is a curve, and its equation can be expressed as follows:

$$
\left\{\begin{array}{l}
y^{2}+z^{2}=\frac{b^{2} f^{2}}{c^{2}}, \\
x= \pm \frac{a e}{c} .
\end{array}\right.
$$

From mathematics, the change rate of the intersection curve (9) with the parameter $a$ can be expressed as

$$
\frac{\mathrm{d} r}{\mathrm{~d} a}=\sqrt{\left(\frac{\mathrm{d} x}{\mathrm{~d} a}\right)^{2}+\left(\frac{\mathrm{d} y}{\mathrm{~d} a}\right)^{2}+\left(\frac{\mathrm{d} z}{\mathrm{~d} a}\right)^{2}} .
$$

Actually, $(\mathrm{d} r / \mathrm{d} a)$ is also the change rate of the hyperboloid along its confocal ellipsoid, which means the change rate of the source location accuracy. The parameter $a$ is the vertex of the hyperboloid, which determines the position of the hyperboloid on the $X$-axis. Also, the term of $(\mathrm{d} r / \mathrm{d} a)$ can be interpreted as the distance that the hyperboloid will move along on the ellipsoid relative to the change of its position on the $X$-axis.

Differentiating equation (9) with respect to $a$ and substituting the partial derivatives into equation (10), we obtain the following equation:

$$
\frac{\mathrm{d} r}{\mathrm{~d} a}=\frac{1}{c} \sqrt{e^{2}+\frac{a^{2} f^{4}}{y^{2}}+\frac{a^{2} f^{4}}{z^{2}}}
$$

Therefore, the density of hyperboloids at any point in the three-dimensional space can be expressed as

$$
\rho_{s}=\frac{c}{\sqrt{e^{2}+\left(a^{2} f^{4} / y^{2}\right)+\left(a^{2} f^{4} / z^{2}\right)}}
$$

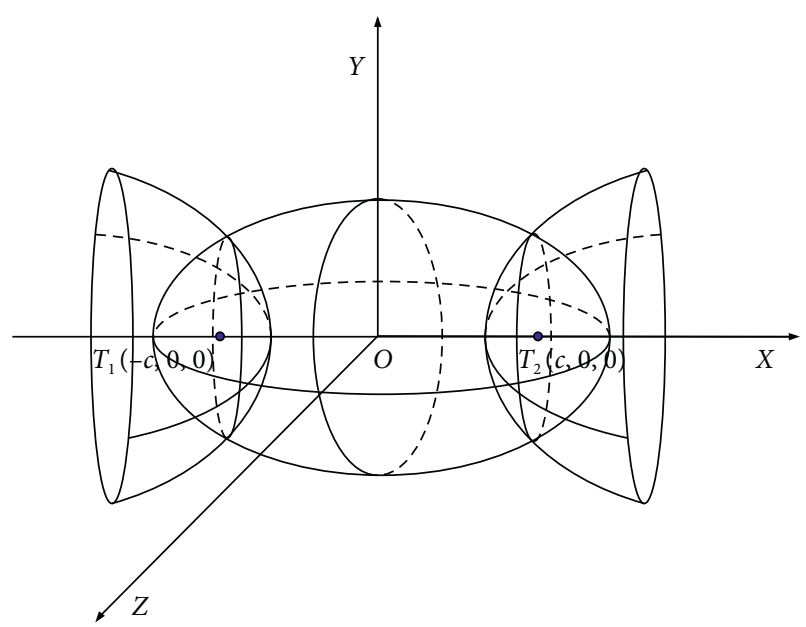

FIgURE 4: The confocal ellipsoid and hyperboloid hyperbola with foci $T_{1}$ and $T_{2}$.

Equations (11) and (12) are the geometrical spreading quantitative formulas in the three-dimensional space. Equation (11) will be degraded as follows in the 2-dimensional plane:

$$
\frac{\mathrm{d} r}{\mathrm{~d} a}=\sqrt{1+\frac{f^{2}}{b^{2}}}
$$

According to the properties of the hyperbola, the larger value of $a$, the further the hyperbola from the central line. So, the term of $(\mathrm{d} r / \mathrm{d} a)$ can be interpreted as the distance that the hyperboloid will move along on the ellipsoid relative to the change of its position on the $X$-axis.

Equation (14) is given based on equation (5) and normalized by parameter $c$ :

$$
\frac{1}{b^{2}}=\frac{1}{1-a^{2}}
$$

The relationship between $\left(1 / b^{2}\right)$ and $a$ is shown in Figure 5. As shown in Figure 5, $\left(1 / b^{2}\right)$ increases with $a$, and this increase rate is accelerated when $a$ approaches $c(c=1)$. So, the term $\left(1 / b^{2}\right)$ represents the influence of the position of hyperbolas. Furthermore, the influence increases dramatically along with the hyperbolas located close to their foci. According to the discussion above, it is understood that the location errors on the hyperbolas (except the intersection point of hyperbolas and $X$-axis) will increase tremendously with the hyperbolas located close to their foci.

As shown in Figure 3, point $K$ is the intersection of the confocal hyperbola and ellipse. Based on the geometry theory, the distance from point $K$ to the origin point $O$ can be calculated as follows:

$$
d=\sqrt{x^{2}+y^{2}}=\sqrt{a^{2}+f^{2}} .
$$

According to equation (15), the value of $d$ only depends on $f^{2}$ once parameter $a$ is determined. The relationship between distance $d$ and parameter $f$ is shown in Figure 6. As illustrated in Figure 6, the value of $d$ increases with the 


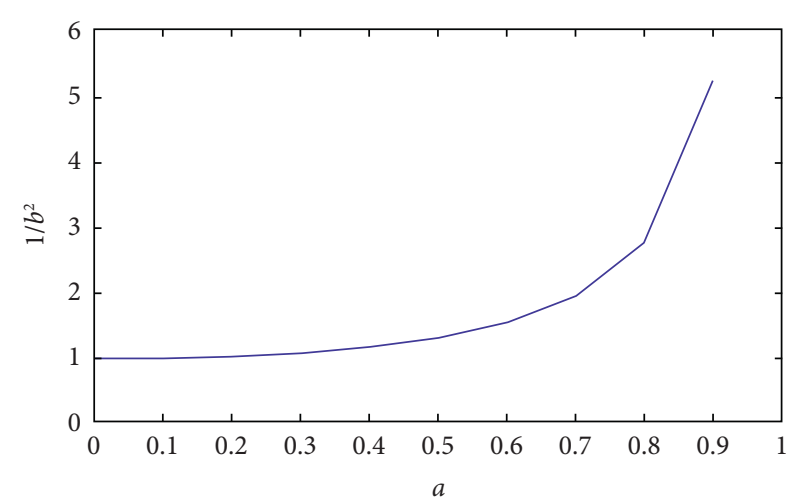

Figure 5: The relationship between $\left(1 / b^{2}\right)$ and $a(c=1)$.

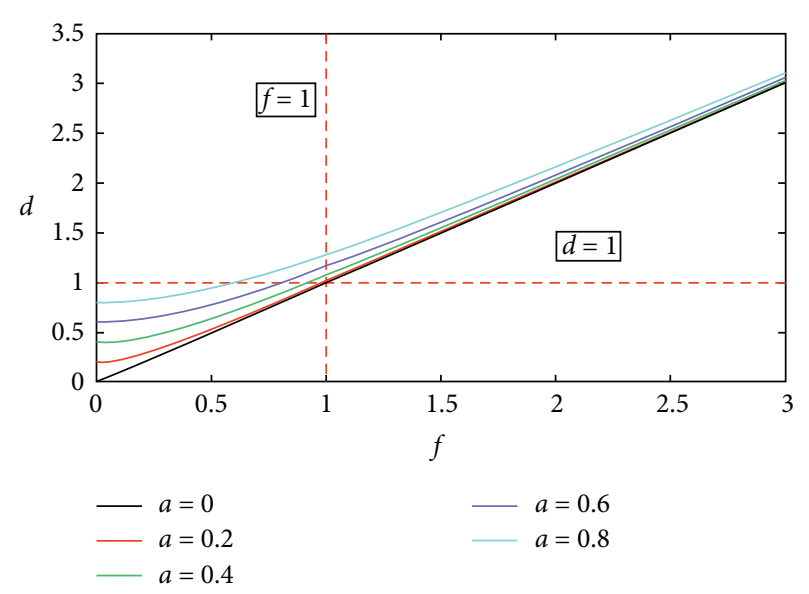

Figure 6: The relationship between $d$ and $f(c=1)$.

increase of the parameter $f$, and $d$ increases almost linearly with a slope of 1 when $f$ is greater than 1 .

Based on the abovementioned analysis, the change of any point in the hyperbolic field is determined by the distance from the origin and the position of its associated hyperbola. The elliptic equation (6) will be transformed into a straight line when $f=0$, and this line is the $X$-axis. The change rate of hyperbolas along the $X$-axis is a constant and is equal to 1 .

Besides the effect of the decreasing density as the sources move away from the center of the sensor network, what is more important is that the density of hyperbolas is uneven spreading. Generally speaking, the density of hyperbolas in the center of the sensor network is higher than the density of those at the edge or outside of network. Thus, the location error of the sources in the center is much smaller than that of the sources at the edge or outside of the network. Based on the characteristic of the uneven geometrical spreading, the location error increases with a nonlinear trend as the sources move away from the center of the network. Also, when the sources are outside of the network, the location error increases sharply because the density of hyperbolas decreases sharply. The geometrical spreading effect shows that it is impossible to get an accurate location when a source is outside of the network. The uneven spreading effect reveals the importance of the relative position of a source and a sensor network.
3.2. Directional Control Effect of the Sensor Network. MS/AE monitoring tests in the field and laboratory both show that the source location errors in different directions are also different. For example, the location error in the Zdirection (vertical direction) is usually much larger than the errors in the $X$ - and $Y$-direction (horizontal plane) during the MS monitoring process in underground mines. So, how the sensor network layout affects the source location in different directions? In the following part, the influence mechanism of the sensor network layout on source location in different directions is studied mathematically, which will explain why the accuracy of location in different directions is different.

For the hyperboloid equation (7), based on the derivative rule of the implicit function, the change rate of any point in the $X$-direction due to the change of the position of a certain hyperboloid can be obtained as

$$
\frac{2 x a^{2}(\mathrm{~d} x / \mathrm{d} a)-2 a x^{2}}{a^{4}}-\left(y^{2}+z^{2}\right)(-1) \frac{(-2 a)}{\left(c^{2}-a^{2}\right)^{2}}=0 .
$$

The density of hyperboloids in the $X$-direction can be calculated as

$$
\rho_{s x}=\frac{\mathrm{d} a}{\mathrm{~d} x}=\frac{x a}{\left(\left(a^{4}\left(y^{2}+z^{2}\right) /\left(c^{2}-a^{2}\right)^{2}\right)+x^{2}\right)} .
$$

Similarly, the density of hyperboloids in the $Y$ - and $Z$ direction can be expressed as follows:

$$
\begin{gathered}
\rho_{s y}=\frac{\mathrm{d} a}{\mathrm{dy}}=\frac{-y\left(c^{2}-a^{2}\right)}{\left(\left(x^{2}\left(c^{2}-a^{2}\right)^{2} / a^{3}\right)+a\left(y^{2}+z^{2}\right)\right)}, \\
\rho_{s z}=\frac{\mathrm{d} a}{\mathrm{~d} z}=-\frac{z\left(c^{2}-a^{2}\right)}{\left(\left(\left(x^{2}+z^{2}\right)\left(c^{2}-a^{2}\right)^{2} / a^{3}\right)+y^{2} a\right)} .
\end{gathered}
$$

Equations (19)-(21) are the quantitative solution formulae of the directional control effect. The equations of the density of hyperboloids in the $Y$ - and $Z$-direction have similar expressions, but the equation of the density of hyperboloid in the $X$-direction is totally different. This is because there are two sensors in the $X$-direction which forms a pair of sensors, while there are no paired sensors on the $Y$ and $Z$-direction (Figure 4). The source location accuracy in the $X$-direction is much higher than that in the $Y$ - and $Z$ direction.

For a two-dimensional source location, it is very difficult to have a good location accuracy in the $Y$-direction when all sensors are located on the $X$-axis. As shown in Figure 7, three sensors are deployed on the $X$-axis. According to the geometrical spreading effect, the location accuracy is closely related to the mesh size and distribution in the hyperbolic field. The smaller the mesh size is, the higher the location accuracy is. Based on the directional control effect, the source location accuracy in different directions can be estimated by the shape and the size of the mesh in the 


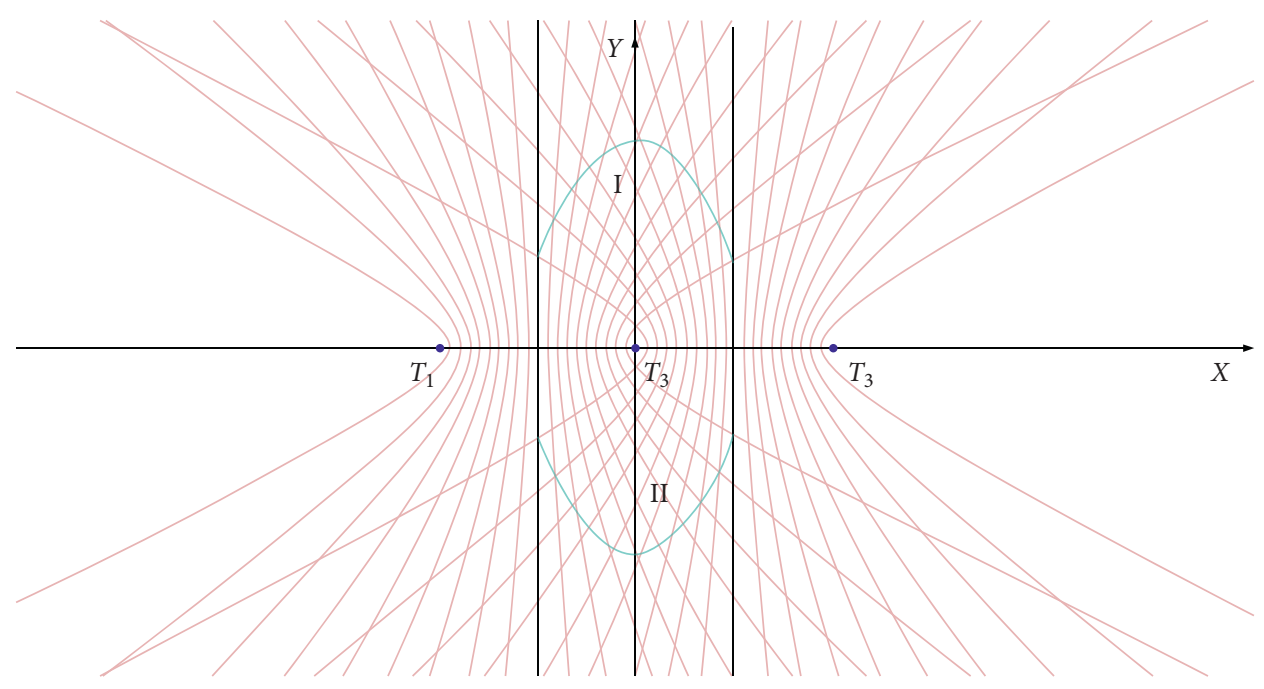

Figure 7: Hyperbolic field associated with three sensors located in the $X$-axis.

hyperbolic field. For the area between the sensors $T_{1}$ and $T_{3}$, except for the area I and II, most of the meshes are rhomboidal in shape with the long diagonal line almost parallel to the $Y$-axis, which clearly shows that the location accuracy in the $X$-direction is much higher than the accuracy in the $Y$-direction. In addition, the ratio of the length and the width of these meshes becomes larger and larger as the source moves away from the $X$-axis, which indicates that the location accuracy in the $Y$-direction decreases faster than that in the $X$-direction.

The direction control effect clearly shows that a source location with good accuracy in all directions can be obtained only when sensors are deployed on all directions. So, it is clear that a sensor network should not be "dimension-reduced." For example, compared to the 3-dimensional underground mining space, most sensors in underground mines are deployed on a near horizontal plane, and these networks are vertical-dimension-reduced ( $Z$-direction-reduced). As a result, the source location accuracy control in the direction ( $Z$-direction) perpendicular to the horizontal plane will be very poor. This is why the vertical location errors are always larger than the horizontal location errors for MS monitoring projects in mines. Therefore, the sensors should be arranged in all directions ( $X$-direction, $Y$-direction, and $Z$-direction) if the monitoring object is a 3 -dimensional space structure.

\subsection{Propagation Characteristics of the Velocity Error and} Arrival Time Error. It is generally known that the AE/MS source location accuracy is mainly affected by the sensors coordinates and network layouts, wave velocity model, arrival time, and source location methods. Among the abovementioned influence factors, sensors coordinates, velocity model, and arrival time are the input parameters. According to the abovementioned discussion, the sensor network layout itself does not induce any errors during the source location process, and it merely transfers the input data errors (such as sensor coordinate, velocity, and arrival time errors) already existing. With the intelligent instruments, the accuracy of sensor coordinates can be as high as $0.2 \times 10^{-6} \mathrm{~mm}$. Thus, the error of coordinates is normally negligible. But, the velocity and arrival time errors are inevitable. Therefore, it is important to study how these velocity and arrival time errors transfer to the final location errors.

According to the hyperbolic governing equation of the source location, the shape of the hyperbola of the source location just depends on the product of wave velocity and the difference of the arrival time. For convenience, this product is denoted as a signal element, namely,

$$
g=2 a_{i j}=v\left|t_{j}-t_{i}\right| \cdot
$$

If there is total initial error $\delta g$, the source will be wrongly located on the adjacent hyperbola with focal length $(d+\delta g)$. If the sensor coordinate error is negligible, then, $\delta g$ is only affected by the arrival time error and velocity error. If there is only arrival time error $\delta t$, according to equation (20), the total initial error is

$$
\delta g_{t}=v \delta t
$$

Equation (21) clearly demonstrates that $\delta t$ is always amplified $v$ times, where $v$ is the wave velocity. Also, if the velocity is a constant, the total errors is not affected by the position of a source when the arrival time error is a primary source of initial error.

If there is only wave velocity error $\delta v$, it is clear that the total initial error is as follows:

$$
\delta g_{v}=\delta v \mathrm{~d} t,
$$

where $\mathrm{d} t$ is the arrival-time difference of two sensors and $\mathrm{d} t=\left|t_{j}-t_{i}\right|$.

It is clear from equation (22) that the total error affected by the velocity error is different from the arrival time error. The velocity error is amplified by $\mathrm{d} t$. Because the arrival-time difference $\mathrm{d} t$ is highly affected by the hyperbola where the source is, the total initial error depends on the position of the source and only velocity errors are under consideration. 
The arrival-time difference is denoted as the "critical arrival-time difference $\left(\mathrm{d} t_{c}\right)$ " when the total error caused by the arrival time error equals to total error caused by the velocity error, i.e., $\delta g_{v}=\delta g_{t}$. The hyperbola calculated by the critical arrival-time difference is called the critical hyperbola. As illustrated in Figure 8, the monitoring area is divided into two parts by the critical hyperbola. Based on equation (21), the location errors are mainly caused by the arrival time error in part I, but the velocity error has the major influence on source location accuracy in part II. The ratio of the area II and area I is denoted as error coefficient $k$. For a square area with a length of 1000 meters, the ratio of area II and I is about 12, which means that the source location error is mainly controlled by the velocity error in most monitoring areas.

The critical hyperbola divides the monitoring area into two regions. Generally speaking, the arrival time error has the major influence on source location in the central area. But, the wave velocity error becomes the leading factor that affects the source location error as the sources move away from the central area. Based on the concept of the critical arrival-time difference, the error coefficient $k$ is getting smaller and smaller with the reduction of the distance between two sensors. For example, $k$ will drop to $9,5,2.5$, and 1.5 if the distance of two sensors reduces to $800,500,300$, and 200 meters. So, the source location errors of most monitoring areas are mainly affected by the velocity errors in field MS monitor project because the distance of any two sensors is usually greater than $200 \mathrm{~m}$. In contrast, the $\mathrm{AE}$ source location errors are mainly controlled by the arrival time error in the laboratory rock AE location tests.

\section{Laboratory Testing}

4.1. Experimental System and Specimen Preparation. The experimental system (Figure 9) consists of the 32-channel AE workstation form Physical Acoustics Company (PAC) and the 5058PR high-voltage pulse generator from Olympus Company. The 5058PR high-voltage pulse generator is designed for the ultrasonic detection of high attenuation material such as rock and coal, which can generate highvoltage ultrasonic signals. $100 \mathrm{~V}$ pulse signals are generated by 5058PR as the known AE sources in this test. The R15 AE sensors from PAC are used to record the AE signals. The resonant frequency of $\mathrm{R} 15$ is $150 \mathrm{kHz}$, and its operating frequency is from 150 to $400 \mathrm{kHz}$. The sampling rate is set as $10 \mathrm{MHz}$.

The granite and marble with high homogeneity were used and cut into a plate and cuboid. The thin plate specimen (GP-1) is granite, and its size is $400 \mathrm{~mm} \times 250 \mathrm{~mm} \times 50 \mathrm{~mm}$ (Figure 11). The cuboid specimen (MP-2) is marble, and its size is $250 \mathrm{~mm} \times 200 \mathrm{~mm} \times 250 \mathrm{~mm}$ (Figure 10). There are five drill holes in the specimen MP-2, which were used to generate internal AE sources during the experiment. The $P$ wave velocity of the specimen GP-1 and MP-2 is $4472.9 \mathrm{~m} / \mathrm{s}$ and $3192.6 \mathrm{~m} / \mathrm{s}$.

4.2. Experimental Program. Two different AE sensor networks were designed by using the GP-1 and MP-2 specimen to study the impacts of the sensor network on the source location. These two different sensor networks are marked as GP-1 sensor network source location and MP-2 sensor network source location experimental program. In order to enhance the coupling effect between the AE sensor and the specimen, the AE sensors are fixed on the specimen by using the tailor-made AE sensor mounting device, and the special coupling agent for AE test is used as well. In addition, the coupling quality of AE sensors is tested by the pencil lead breaking before the formal experiments.

4.2.1. GP-1 Sensor Network Source Location Experimental Program. As shown in Figure 11, the AE sensor network consists of 12 AE sensors in GP-1 experimental program. There are $29 \mathrm{AE}$ pulse sources on the thin plate specimen. The coordinates of the sensors and sources are given in Tables 1 and 2 .

4.2.2. MP-2 Sensor Network Source Location Experimental Program. As illustrated in Figure 10, the AE sensor network consists of $18 \mathrm{AE}$ sensors in MP-2 experimental program. According to experimental requirements, $5 \mathrm{AE}$ sources are located in the drilling holes, and $16 \mathrm{AE}$ sources are located on the surface of the cuboid. The coordinates of the sensors and sources are given in Tables 3 and 4 .

4.3. Analysis of the AE Source Location. The simplex method and L1-norm were used to calculate the AE source in the test [9]. The $29 \mathrm{AE}$ source locations in GP-1 experimental program and 21 source locations in MP-2 experimental program were calculated. The location errors of each $\mathrm{AE}$ source in the GP-1 and MP-2 sensor network are shown in Figure 12.

As clearly shown in Figure 12, the location errors are, in general, the smallest when the sources are located at the center of the sensor network. The location accuracy decreases with a nonlinear downtrend as the source moves away from the center of the network. For the test GP-1, the seven sources at the center of the network have the highest horizontal location accuracy, and the horizontal location errors are less than $8 \mathrm{~mm}$. However, the location error increases to around $20 \mathrm{~mm}$ when the source is outside the hexagon of sensors on the upper surface of sample GP-1. The sources in the five boreholes of test MP-2 have the highest location accuracy, and their errors are only $2 \sim 4 \mathrm{~mm}$. But, the location error of the sources at the surface of the sample is up to $30 \mathrm{~mm}$.

According to the directional control effect, it is a dimension-reduced ( $Z$-direction) sensor network in test GP-1, and as a result, it is very hard to have an accurate location in the $Z$ direction because all the sensors are arranged nearly horizontally. The source location results in Figure 12 clearly show that the location error of each source, whether inside or outside the network, in the $Z$-direction is much larger than the location error in the horizontal direction ( $X$-direction or $Y$-direction) in the GP-1 test. Thus, the total location error is mainly affected by the $Z$-direction error. The horizontal location errors $(X$ - 


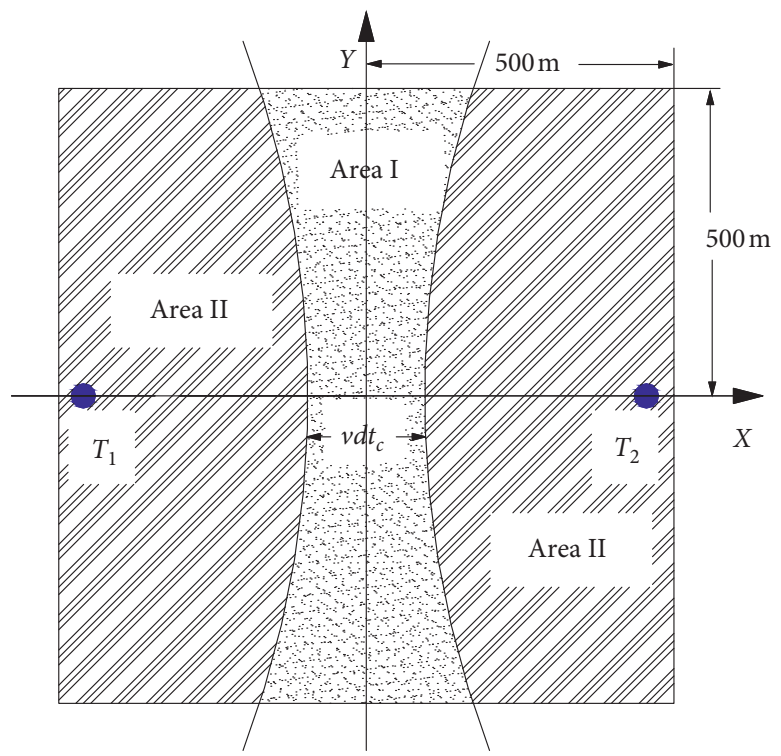

FIGURE 8: The areas controlled by the arrival time error and wave velocity error.

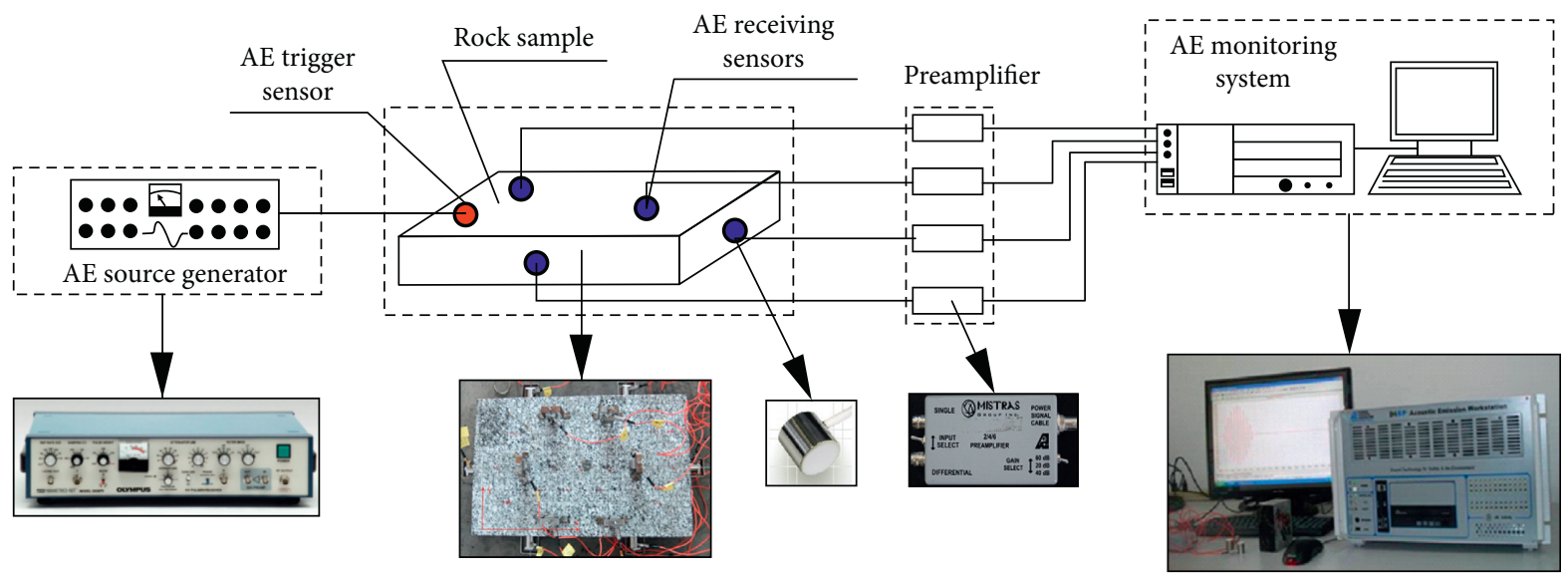

FIGURE 9: Sketch of the experimental system of the sensor network and AE source location test.

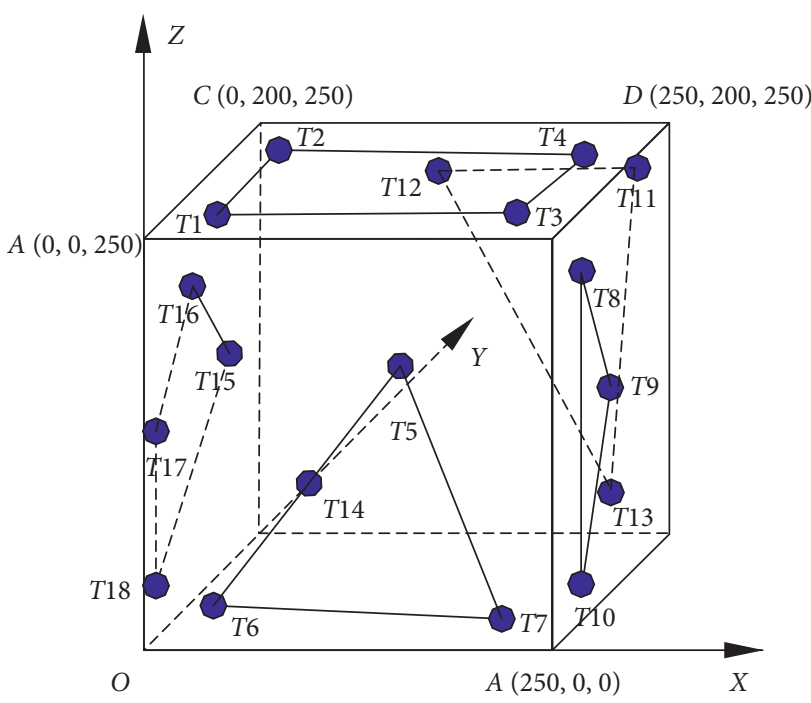

(a)

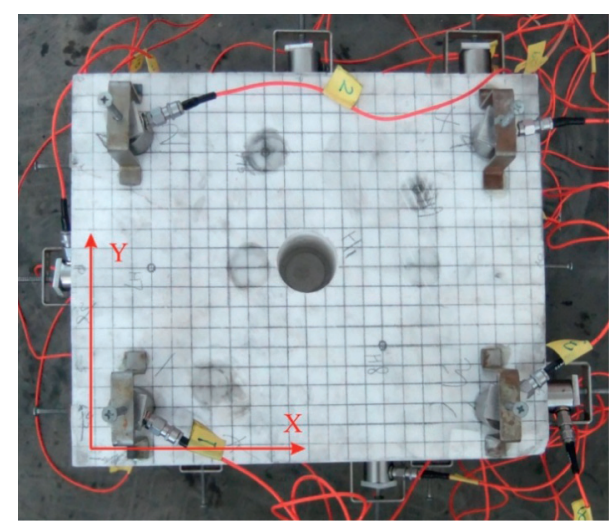

(b)

Figure 10: The AE sensor network layout for the specimen GP-1, (a) is the sketch map, (b) is the physical photo. 
TABLE 1: The coordinates of sensors on the specimen GP-1.

\begin{tabular}{lccc}
\hline Sensors & $X(\mathrm{~mm})$ & $Y(\mathrm{~mm})$ & $Z(\mathrm{~mm})$ \\
\hline T1 & 100 & 249 & 28 \\
T2 & 290 & 248 & 28 \\
T3 & 104 & 0 & 30 \\
T4 & 298 & 0 & 27 \\
T5 & 100 & 120 & 50 \\
T6 & 151 & 32 & 50 \\
T7 & 260 & 29 & 50 \\
$T 8$ & 300 & 122 & 50 \\
$T 9$ & 251 & 205 & 50 \\
T10 & 150 & 210 & 50 \\
T11 & 0 & 129 & 27 \\
T12 & 398 & 120 & 28 \\
\hline
\end{tabular}

TABLE 2: The coordinates of the pulse sources on the specimen GP1 .

\begin{tabular}{|c|c|c|c|}
\hline Sources & $X(\mathrm{~mm})$ & $Y(\mathrm{~mm})$ & $Z(\mathrm{~mm})$ \\
\hline$S 1$ & 200 & 120 & 50 \\
\hline$S 2$ & 170 & 160 & 50 \\
\hline$S 3$ & 150 & 120 & 50 \\
\hline$S 4$ & 170 & 180 & 50 \\
\hline S5 & 230 & 80 & 50 \\
\hline S6 & 250 & 120 & 50 \\
\hline S7 & 230 & 160 & 50 \\
\hline$S 8$ & 200 & 210 & 50 \\
\hline$S 9$ & 120 & 160 & 50 \\
\hline$S 10$ & 120 & 80 & 50 \\
\hline$S 11$ & 200 & 30 & 50 \\
\hline$S 12$ & 280 & 80 & 50 \\
\hline$S 13$ & 280 & 160 & 50 \\
\hline S14 & 70 & 180 & 50 \\
\hline$S 15$ & 40 & 120 & 50 \\
\hline$S 16$ & 70 & 60 & 50 \\
\hline$S 17$ & 330 & 60 & 50 \\
\hline$S 18$ & 360 & 100 & 50 \\
\hline$S 19$ & 330 & 180 & 50 \\
\hline$S 20$ & 60 & 0 & 30 \\
\hline$S 21$ & 200 & 0 & 30 \\
\hline$S 22$ & 340 & 0 & 30 \\
\hline$S 23$ & 340 & 250 & 30 \\
\hline$S 24$ & 200 & 250 & 30 \\
\hline$S 25$ & 60 & 250 & 30 \\
\hline S26 & 0 & 180 & 30 \\
\hline$S 27$ & 0 & 60 & 30 \\
\hline$S 28$ & 400 & 180 & 30 \\
\hline$S 29$ & 400 & 60 & 30 \\
\hline
\end{tabular}

direction and $Y$-direction location errors) are further studied to verify the geometrical spreading effect. The contour map of location errors on the $X$-direction and $Y$-direction is plotted in Figure 13. The solid blue circles represent sensors. The deeper the red color is, the higher the location accuracy is. The location accuracy rate can be represented by the density of the contour lines. Also, the higher the contour density, the faster the location accuracy decreases.

As illustrated in Figure 13, the density of the location error contour varies at different points. Usually, the density of the location error contour in the center area is smaller than that at the edge or outside of the sensor network, which means that the decline rate of the location error is getting faster and faster as the source moves away from the center of the sensor network. In other words, the location error increases with a nonlinear growth trend as the source moves away from the center of the network.

The distribution law of the location error in the $X$ direction and $Y$-direction is different from each other. As shown in Figure 13, the location error in the $X$-direction is mainly affected by two subnetworks: a rectangular subnetwork composed of T1 T4 sensors and a orthohexagonal subnetwork composed of T5 T10 sensors. Also, the location error in the $Y$-direction is mainly affected by three subnetworks, namely, a orthohexagonal subnetwork composed of T5 T10 sensors and two triangular subnetworks composed of T6, T10, T11 and T7, T9, T12. The location errors in both the $X$-direction and $Y$-direction of the sources located in the corners of the rectangle (black circle areas) are very large and out of control because these four corners are outside of the sensor network. So, we will get a more optimized network configuration if the sensors $T 1, T 2, T 3$, and $T 4$ need move to the four corners of the rectangle.

As can be seen from Tables 1 and 2, the distances from the edge to the center of the sensor network are about $80 \mathrm{~cm}$ and $50 \mathrm{~cm}$ in the $X$-direction and $Y$-direction, respectively, in the GP-1 test. As can be seen from Tables 3 and 4, the distances from the edge to the center of the sensor network are about $90 \mathrm{~cm}$ in all directions in the MP-2 test. The relationship between the location error in different directions and the distance from the source to the center of the network is shown in Figure 14. When the source is inside the sensor network, the location error in each direction increases slowly with the increase in distance from the center of the network. But, when the source is outside the sensor network, the location error in every direction increases sharply with the increase in distance. There is an inflection point that the increasing rate of the location error increases significantly when the distance exceeds this point. As shown in Figure 14, the inflection points are $50 \mathrm{~cm}$ and $80 \mathrm{~cm}$ in the $X$-direction and $Y$-direction, respectively, in the GP-1 test and are $90 \mathrm{~cm}$ in all directions in the MP-2 test. According to Tables 1 4, this infection point is just located on the edge of the sensor network.

The wave velocity error is an important type of input data error during the source location process. Here, we assume different errors in the velocity model and relocate the sources by using velocities with different errors. The calculated results are illustrated in Figure 15. The location errors increase with the increase in the velocity error. For the sources inside and outside the network, the location errors caused by the same velocity error are completely different. For example, if $10 \%$ error is involved in the velocity, the location error is about $20 \mathrm{~mm}$ when the sources are inside the network, but the location error will reach $300 \mathrm{~mm}$ when the sources are outside the network. In practice, the velocity error is usually within $10 \%$, which means that the location errors caused by the velocity error are acceptable if the sources are located in the sensor network. 


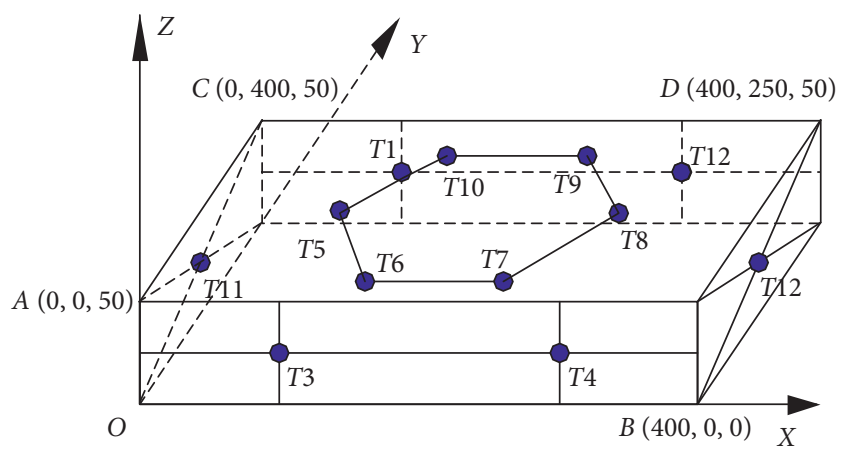

(a)

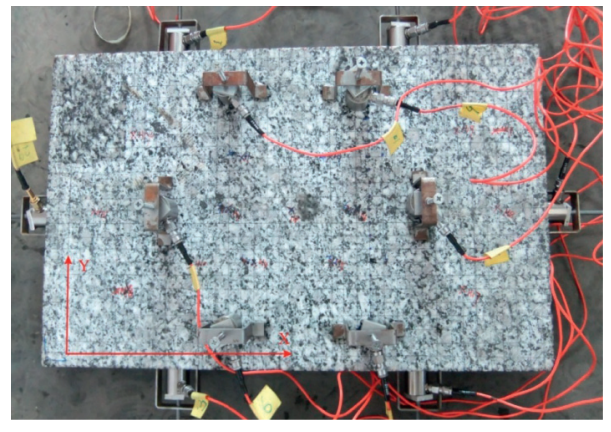

(b)

FIgure 11: The AE sensor network layout for the specimen MP-2: (a) the sketch map and (b) the physical photo.

TABLE 3: The coordinates of sensors on the specimen MP-2.

\begin{tabular}{lccc}
\hline Sensors & $X(\mathrm{~mm})$ & $Y(\mathrm{~mm})$ & $Z(\mathrm{~mm})$ \\
\hline$T 1$ & 30 & 30 & 250 \\
$T 2$ & 31 & 168 & 250 \\
$T 3$ & 225 & 28 & 250 \\
$T 4$ & 219 & 165 & 250 \\
$T 5$ & 162 & 0 & 160 \\
$T 6$ & 32 & 0 & 28 \\
$T 7$ & 220 & 0 & 18 \\
$T 8$ & 248 & 30 & 220 \\
$T 9$ & 248 & 112 & 111 \\
$T 10$ & 248 & 30 & 28 \\
$T 11$ & 221 & 202 & 220 \\
$T 12$ & 120 & 202 & 218 \\
$T 13$ & 218 & 202 & 30 \\
$T 14$ & 29 & 201 & 20 \\
$T 15$ & 0 & 180 & 130 \\
$T 17$ & 0 & 102 & 190 \\
$T 17$ & 0 & 20 & 132 \\
$T 18$ & 0 & 30 & 30 \\
\hline
\end{tabular}

\section{Discussion}

The MS monitoring area is a semiopen three-dimensional space in underground mines. The spatial form and scale and rock properties of the monitoring area are very complicated. In addition, the installation position of the sensor is also limited by the field conditions and coupled with the economic considerations, and the number of sensors in the monitoring system cannot be increased indefinitely. Therefore, it is of great practical significance to establish an optimal sensor network with the least number of sensors. Some principles of optimizing sensor network are given based on the mechanism of sensor network in the control of source location.

As shown in Figure 16, it is assumed that the main MS monitoring area is around $-500 \mathrm{~m}$ level. Four different pairs of MS sensors are illustrated in Figure 16, and the vertical drop of every paired sensors is 200 miters. The two sensors in Figures 16(a), 16(c), and 16(d) are located on both sides of the $-500 \mathrm{~m}$ level, and the vertical distance of the two sensors to the $-500 \mathrm{~m}$ level is equal. However,
TABLE 4: The coordinates of the pulse sources on the specimen MP2.

\begin{tabular}{lccc}
\hline Sources & $X(\mathrm{~mm})$ & $Y(\mathrm{~mm})$ & $Z(\mathrm{~mm})$ \\
\hline$S 1$ & 120 & 100 & 170 \\
$S 2$ & 55 & 44 & 190 \\
$S 3$ & 170 & 87 & 70 \\
$S 4$ & 190 & 130 & 180 \\
$S 5$ & 80 & 160 & 110 \\
$S 6$ & 100 & 160 & 250 \\
$S 7$ & 40 & 100 & 250 \\
$S 8$ & 160 & 60 & 250 \\
$S 9$ & 180 & 140 & 250 \\
$S 10$ & 190 & 200 & 80 \\
$S 11$ & 140 & 200 & 130 \\
$S 12$ & 30 & 200 & 160 \\
$S 13$ & 250 & 130 & 200 \\
$S 14$ & 250 & 180 & 120 \\
$S 15$ & 250 & 50 & 120 \\
$S 16$ & 210 & 0 & 210 \\
$S 17$ & 120 & 0 & 70 \\
$S 18$ & 20 & 0 & 210 \\
$S 19$ & 0 & 170 & 220 \\
$S 20$ & 0 & 30 & 220 \\
$S 21$ & 0 & 150 & 30 \\
\hline
\end{tabular}

the difference is that the angle between the connection line of the two sensors and the horizontal plane in the three cases is $90^{\circ}, \alpha$ and $\beta$, respectively. Also, the relationship of the three angles satisfies the following equation: $\alpha<\beta<90^{\circ}$. According to the directional control effect, the case of Figure 16(a) has the highest source location accuracy, the case of Figure 16(c) has the second highest, and the case of Figure 16(d) has the lowest accuracy in the vertical direction. Here, we define the angle between the connection line of the two sensors and the horizontal plane as the direction angle. Thus, when the sensors are located on both sides of the monitoring plane, the larger the direction angle is, the higher is the location accuracy in the direction perpendicular to the monitoring plane.

Although the direction angles of Figures 16(a) and 16(b) are the same, the relative positions of sensors and monitoring level 


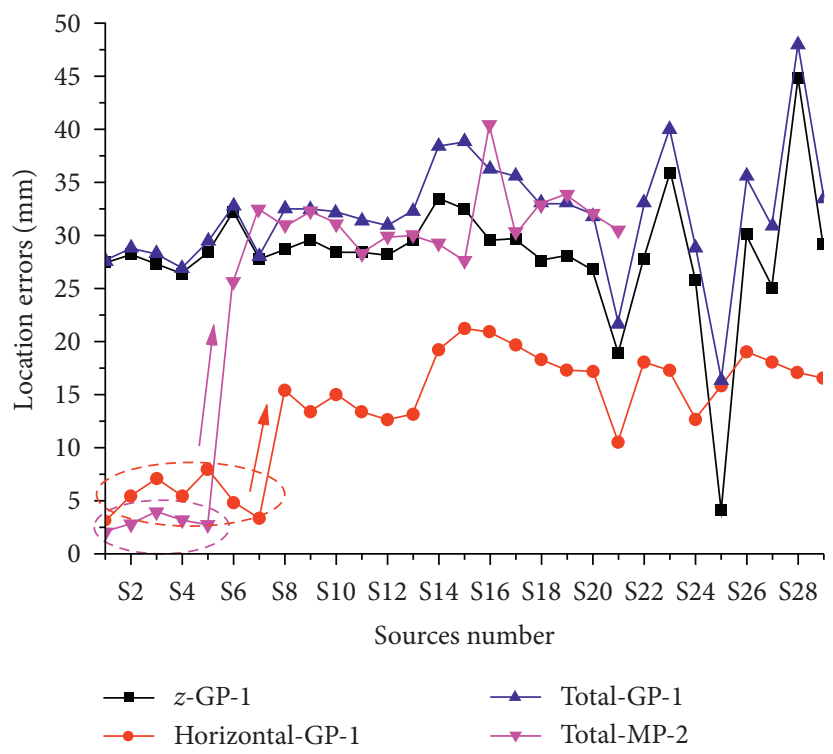

FIgURE 12: AE source location errors of the sample GP-1 and MP-2.

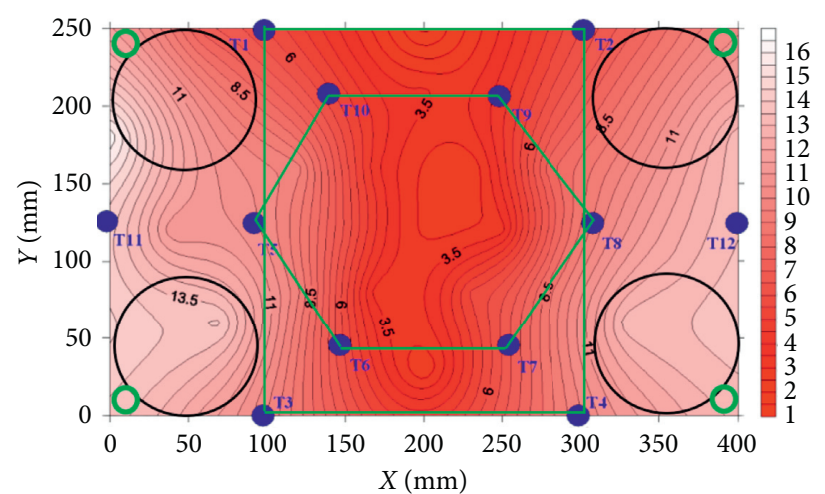

(a)

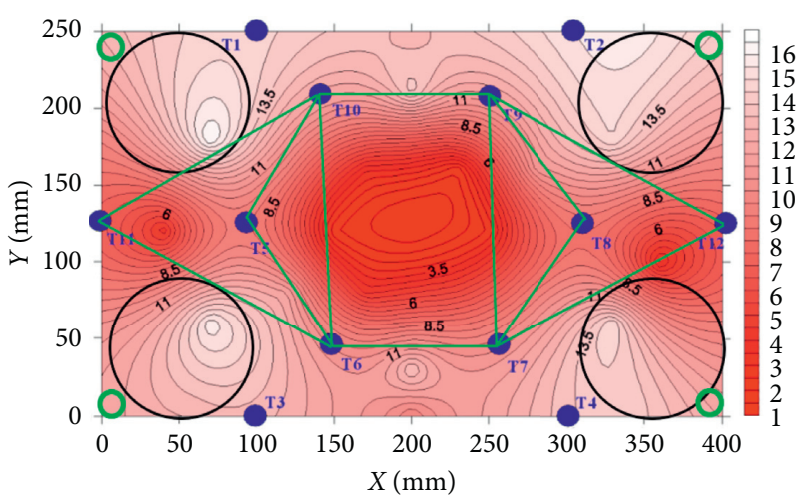

(b)

FIgURE 13: The contour map of AE source location errors on the $X$ - and $Y$-direction of the sample GP-1. (a) $X$-direction. (b) $Y$-direction.

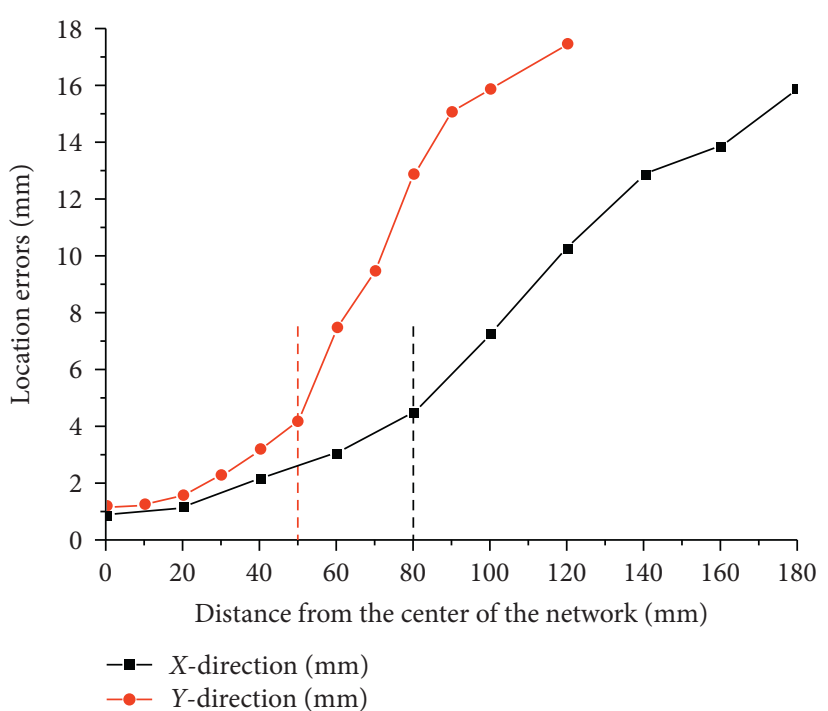

(a)

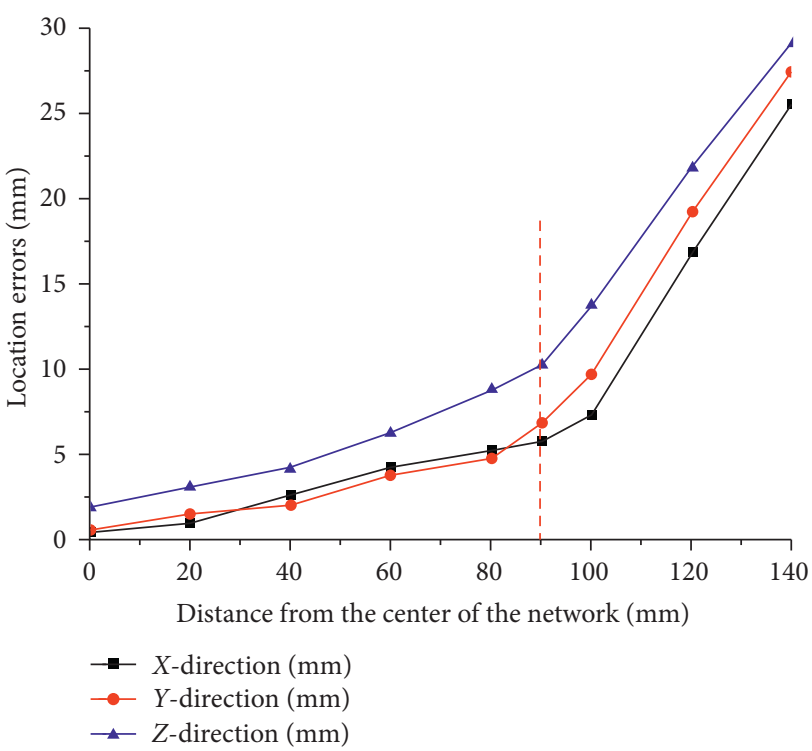

(b)

FIGURE 14: Relationship between the location errors and the distance from the center of the network. (a) GP-1. (b) MP-2. 


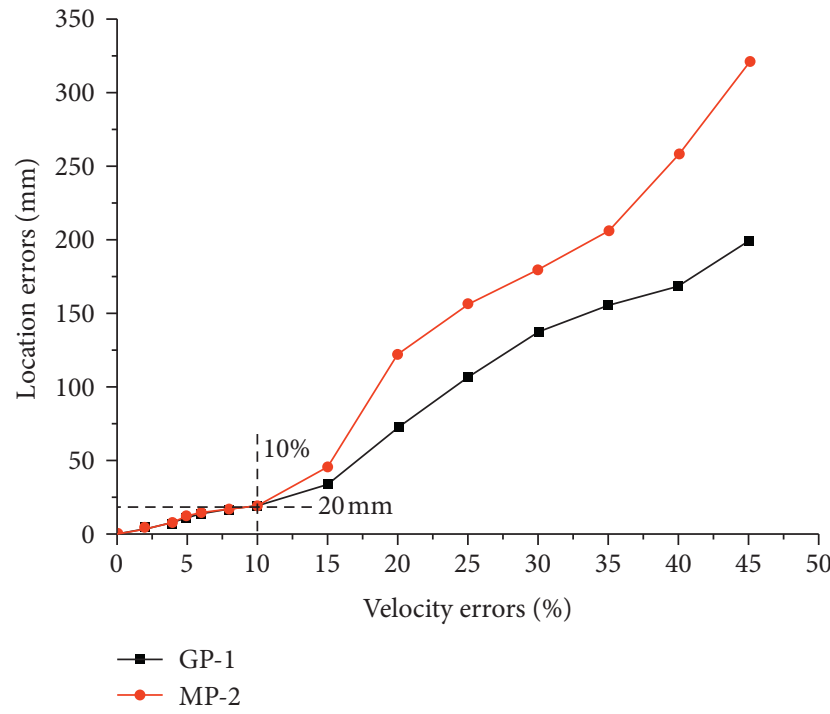

(a)

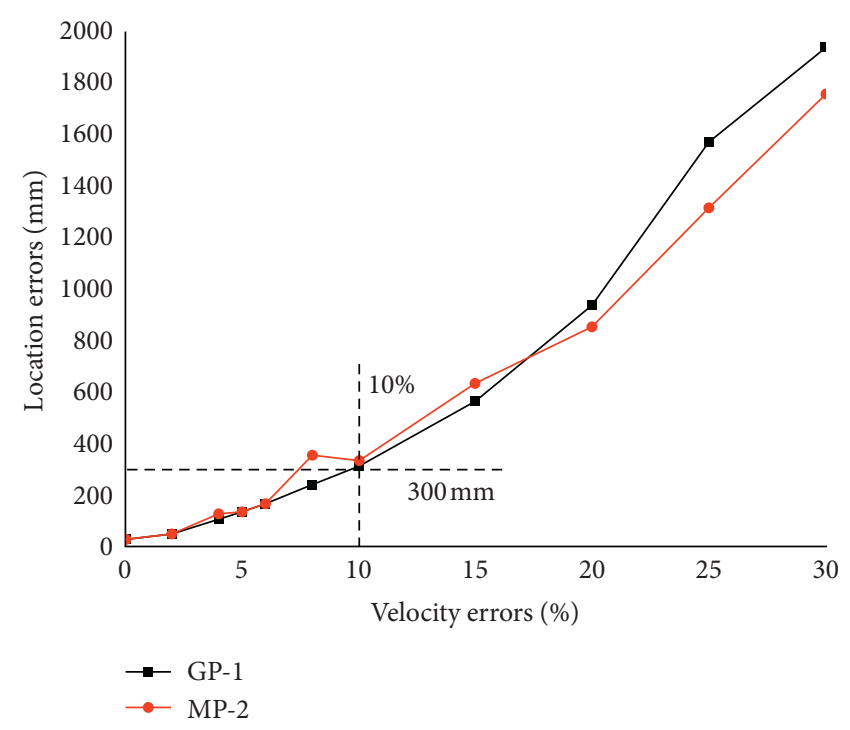

(b)

FIGURE 15: Relationship between location errors and velocity errors. (a) Inside of the sensor network. (b) Outside of the sensor network.

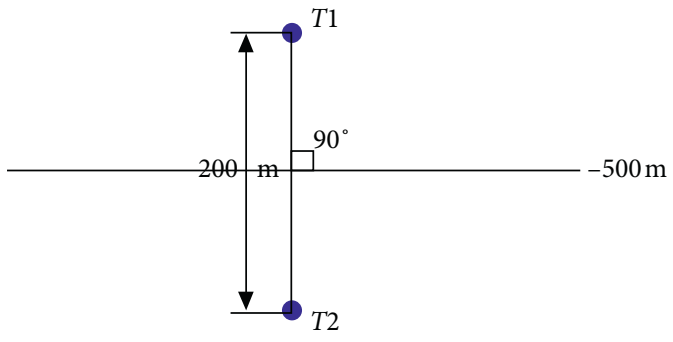

(a)

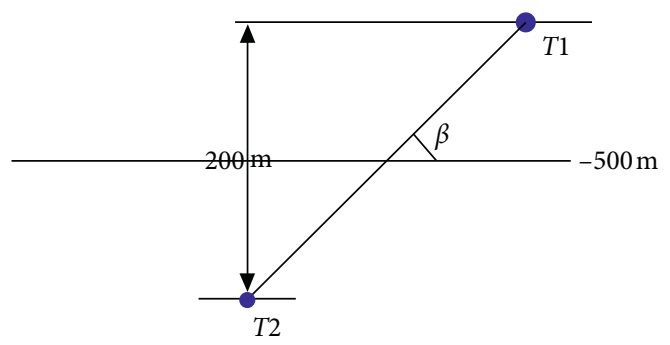

(c)

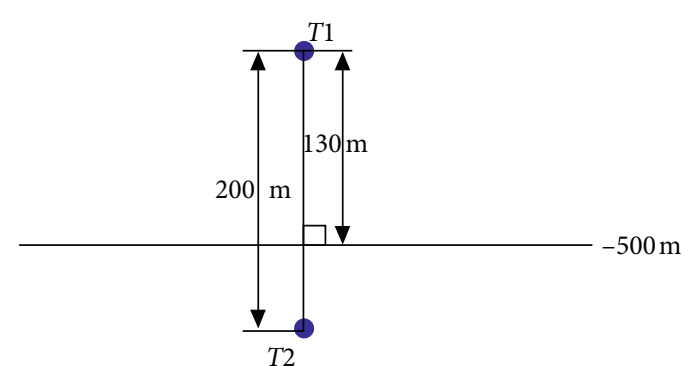

(b)

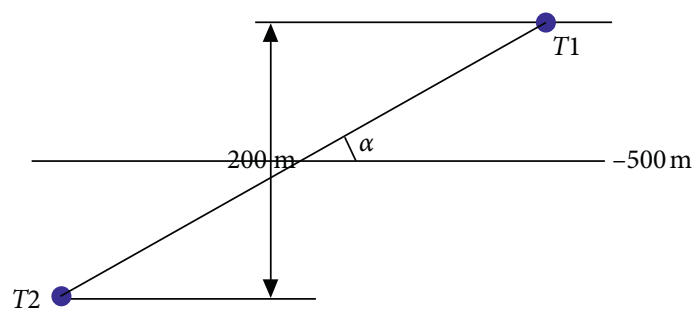

(d)

FIGURE 16: Four typical paired sensors at $-500 \mathrm{~m}$ monitoring level.

are different. The monitoring plane vertically bisects the connection line between the two sensors in Figure 16(a). But, in Figure 16(b), the monitoring plane vertically passes through one third of the connection line between the two sensors. According to the geometrical spreading effect, the location accuracy in the vertical direction of the case in Figure 16(b) is less than that in Figure 16(a).

In addition, the whole monitoring area should be considered when designing the sensor network. The sensor network should surround the entire monitoring area. As illustrated in Figure 17, the whole MS monitoring area is the rectangle surrounded by points $A, B, C$, and $D$. Although the direction angle $w>y$ and $x>z$ in Figure 17, the sensor network in Figure 17(a) does not surround the whole monitoring area. The shadow part is located outside the sensor network, so the location accuracy of the shadow part cannot be guaranteed. However, the sensor network completely surrounds the whole monitoring area in Figure 17(b), so the sensor network layout of Figure $17(\mathrm{~b})$ is more preferable in practice.

According to the abovementioned analysis, combined with the geometrical spreading effect, directional control 


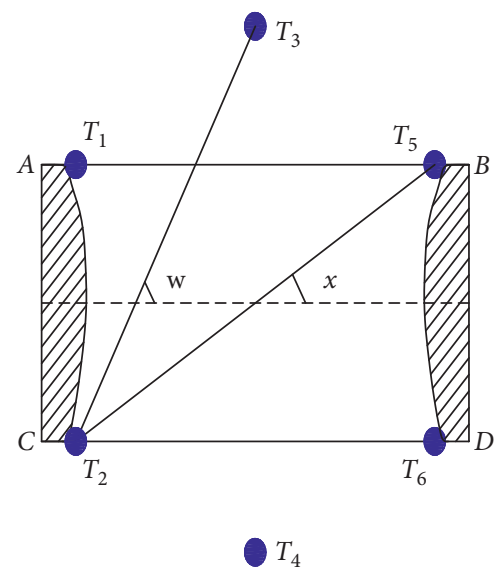

(a)

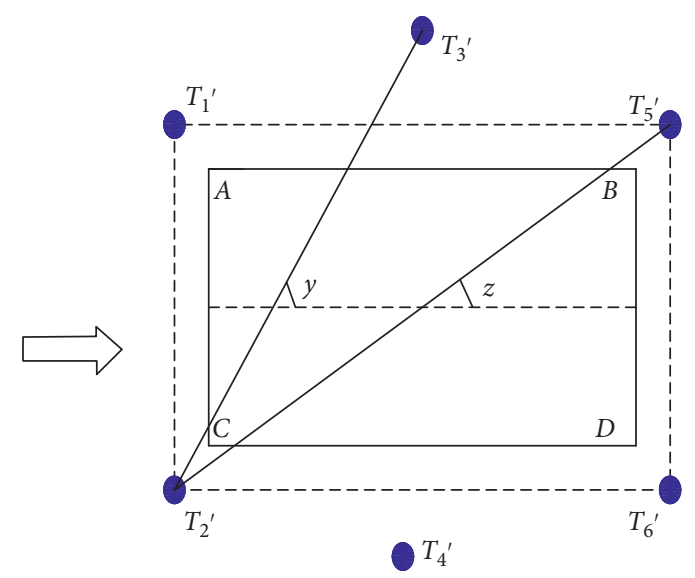

(b)

FIGURE 17: Optimization of the MS monitoring sensor network layout.

effect, and the critical hyperbola, some principles of the sensor network are given as follows:

(1) The sensor network shall surround the main monitoring area in the $X-, Y_{-}$, and $Z$-direction. Also, the sensors should avoid installing on the same straight line, plane, hyperbola, or hyperboloid at the same time.

(2) In consideration of the real monitoring environmental and economic factors, the number of sensors should be increased as much as possible. In addition, we make the sensor network form an irregular spatial layout because this network layout can increase the arrival-time difference of different sensors, thereby reducing the effect of the arrival time error on the source location and improving the source location accuracy.

(3) After the monitoring area and the total number of sensors are determined, the sensors located at the center and edge of the monitoring area should be balanced. In this way, not only the overall location accuracy of the entire monitoring area can be balanced but also the influence of the wave velocity error of the sources located on the edge area of the network and the influence of the arrival time error of the sources located in the center area of the network can be reduced at the same time.

(4) Some sensor subnetworks should be formed based on the spatial characteristics of the monitoring area.

(5) Since the establishment of MS monitoring system is a costly and time-consuming project, it is necessary to consider not only the current requirements but also the long-time monitoring requirements for the MS monitoring when the sensor network is designed.

\section{Conclusions}

(1) Based on the arrival-time-difference principle, the hyperbolic/hyperboloidal governing equations of the source location are established. The path of the potential source determined by two sensors is a hyperbola on the plane and a hyperboloid in the space. The position and the shape of a hyperbola or hyperboloid where the source is located just depend on the arrival-time difference when the velocity model is determined. The sensor network does not induce any errors during the source location process; it only affects the source location accuracy by amplifying the existing errors of the input data such as arrival time and wave velocity. Also, the amplification effect of the input data errors is characterized by nonuniformity because of the nonuniformity of the hyperbolic/hyperboloidal field. This nonuniformity amplification effect is an important reason that the accuracy and stability of source location depend on the relative position of the true source and the sensor network.

(2) According to the governing equation of the source location and the nonuniformity geometrical characteristics of the hyperbolic/hyperboloidal field, the geometrical spreading effect and the directional control effect of the sensor network are revealed, and the three-dimensional quantitative models of these two effects are established. In addition, these two effects are verified by two source location experiments with different AE sensor networks. The geometrical spreading effect indicates that the location accuracy declines when a source moves away from the center of the network. The decline rate of the source location accuracy is small when the source is inside the sensor network. But, the decline rate drops sharply when the source is crossing the edge of the sensor network. Also, this decline rate has nonuniformity characteristics. The directional control effect shows that the source location accuracy of the same source in different directions is different. As the source moves away from the center of the network, there exists a direction that the location 
accuracy in this direction decreases faster than the other directions. In other words, the geometrical spreading effect in different directions is also different.

(3) The influence of the wave velocity error and the arrival time error on the source location accuracy is analytically compared, and the propagation characteristics of these two input data errors during the source location process are revealed. The concepts of the critical arrival-time difference and the critical hyperbola/hyperboloid are proposed. The critical hyperbola/hyperboloid can divide the monitoring area into two different areas where the source location errors are dominated by the arrival time error and the wave velocity error, respectively. Usually, the arrival time errors have the major influence in the central region. However, as the source moves away from the center area of the sensor network, the velocity error gradually becomes the major influence in the remaining area. This study shows that the velocity error is more significant for the large-scale MS monitoring in field. In contrast, the source location error is mainly controlled by the arrival time error in the small-scale laboratory rock AE location test.

(4) The concept of the paired sensors and the direction angle of paired sensors are proposed, and the relationship between the source location and the layout of four typical paired sensors is discussed. When the two sensors are located on both sides of the MS monitoring plane, the larger the direction angle, the higher the source location accuracy in the direction perpendicular to the monitoring plane. An optimal sensor network should surround the potential monitoring area in the three directions of $X, Y$, and $Z$, that is, the monitoring area is best located inside the sensor network. Also, a sensor network should not be dimension-reduced. According to the spatial distribution characteristics of the monitoring area, if the total number of sensors is determined, a more reasonable sensor network will be formed by appropriately reducing the number of sensors in the center area and adding sensors to the periphery of the monitoring area. In addition, the sensor subnetwork should be formed to increase the interference resistance performance of the sensor network and source position.

\section{Data Availability}

All of the important data in the laboratory test used to support the findings of this study are included within the article. Also, the original data are available from the corresponding author upon request.

\section{Conflicts of Interest}

The authors declare that there are no conflicts of interest regarding the publication of this paper.

\section{Acknowledgments}

The authors are grateful to the associate editor and reviewer for their valuable comments, which contributed significantly to the improvement of this paper. This work was supported by the National Natural Science Foundation of China (51874296), the Natural Science Foundation of Jiangsu Province (BK20190080), the China Postdoctoral Science Foundation Grant (2018M640533), and the State Key Laboratory of Coal Resources and Mine Safety of China (SKLCRSM19X002).

\section{References}

[1] R. Hardy, Acoustic Emission/Microseismic Activity, Vol. 1, A. A. Balkema Publishers, Lisse, Netherlands, 2003.

[2] L. J. Dong, W. Zou, D. Y. Sun, X. Tong, X. Li, and W Shu, "Some developments and new insights for microseismic/ acoustic emission source localization," Shock and Vibration, vol. 2019, Article ID 9732606, 15 pages, 2019.

[3] N. Li, E. Y. Wang, and M. C. Ge, "Microseismic monitoring technique and its applications at coal mines: present status and future prospects," Journal of China Coal Society, vol. 42, no. S1, pp. 83-96, 2017.

[4] S. Y. Gong, L. M. Dou, A. Y. Cao et al., "Study on optimal configuration of seismological observation network for coal mine," Chinese Journal of Geophysics, vol. 53, no. 2, pp. 457-465, 2010.

[5] N. Li and E. Y. Wang, Mechanisms of Key Factors for Microseismic Source Location and its Application, China university of mining and technology press, Xuzhou, China, 2015.

[6] L. Geiger, "Probability method for the determination of earthquake epicentres from the arrival time only," Bulletin Saint Louis Univeisity, vol. 8, pp. 60-71, 1912.

[7] Y. Tia and X. F. Chen, "Review of seismic location study," Progress in Geophysics, vol. 17, no. 1, pp. 147-155, 2002.

[8] F. Waldhauser and W. L. Ellsworth, "A double-difference earthquake location algorithm: method and application to the northern Hayward Fault, California," Bulletin of the Seismological Society of America, vol. 90, no. 6, pp. 1353-1368, 2000.

[9] B. R. Chen, X. T. Feng, S. L. Li et al., "Microseismic sources location with hierarchical strategy based on particle swarm optimization," Chinese Journal of Rock Mechanics and Engineering, vol. 28, no. 4, pp. 740-749, 2009.

[10] N. Li, E. Wang, M. Ge, and Z. Sun, "A nonlinear microseismic source location method based on Simplex method and its residual analysis," Arabian Journal of Geosciences, vol. 7, no. 11, pp. 4477-4486, 2014.

[11] M. C. Ge, "Efficient mine microseismic monitoring," International Journal of Coal Geology, vol. 64, no. 8, pp. 44-56, 2005.

[12] R. A. Uhrhammer, "Ananlysis of small seismographic station networks," Bulletin of the Seismological Society of America, vol. 70, pp. 1369-1379, 1980.

[13] M. H. Wirth, R. Blandford, and H. Husted, Estimation of Network Detection and Location Ability, DARPA Order No. 255 1, Teledyne Geotech, Aleksandria, VR, USA, 1978.

[14] S. K. Arora, T. K. Basu, and C. A. Krishnan, "Relative performance of augmented seismic network under random arrival-time errors," Proceedings of the Indian Academy of Sciences, vol. 89, pp. 329-367, 1980. 
[15] A. Kijko, "An algorithm for the optimum distribution of a regional seismic network? I," Pure and Applied Geophysics, vol. 115, no. 4, pp. 999-1009, 1977.

[16] A. Kijko, "An algorithm for the optimum distribution of a regional seismic network? II. An analysis of the accuracy of location of local earthquakes depending on the number of seismic stations: an analysis of the accuracy of location of local earthquakes depending on the number of seismic stations," Pure and Applied Geophysics PAGEOPH, vol. 115, no. 4, pp. 1011-1021, 1977.

[17] A. Kijko and M. Sciocatti, "Optimal spatial distribution of seismic stations in mines," International Journal of Rock Mechanics and Mining Sciences \& Geomechanics Abstracts, vol. 32, no. 6, pp. 607-615, 1995.

[18] C. I. Trifu, "Optimal development of a regional seismic network. Exemplification for Romania," Review Roumaine Physics, vol. 28, pp. 81-90, 1983.

[19] N. Rabinowitz and D. M. Steinberg, "Optimal configuration of a seismographic network: a statistical approach," Bulletin of the Seismological Society of America, vol. 80, no. 1, pp. 187$196,1990$.

[20] Y. X. Xia, J. F. Pan, Y. J. Wang et al., "Study of rule of surrounding rock failure and stress distribution based on highprecision microseismic monitoring," Journal of China Coal Society, vol. 32, no. 2, pp. 239-243, 2011.

[21] L. Z. Tang, C. X. Yang, and C. L. Pan, "Optimization of microseismic monitoring network for large-scale deep well mining," Chinese Journal of Rock Mechanics and Engineering, vol. 25, no. 10, pp. 2036-2042, 2006.

[22] D. M. Steinberg and N. Rabinowitz, "Optimal seismic monitoring for event location with application to on site inspection of the comprehensive nuclear test ban treaty," Metrika, vol. 58, no. 1, pp. 31-57, 2003.

[23] S. Y. Gong, L. M. Dou, X. P. Ma et al., "The method to identify the optimal channel numbers for increasing the location accuracy of microseismic events in coal mine," Journal of China Coal Society, vol. 35, no. 12, pp. 2017-2021, 2010.

[24] A. J. Mendecki, Seismic Monitoring in Mines, Chapman and Hall Press, London, UK, 1997.

[25] B. X. Jia and G. Z. Li, "The research and application for spatial distribution of mines seismic monitoring stations," Journal of China Coal Society, vol. 35, no. 12, pp. 2045-2048, 2010.

[26] H. T. Wang, W. X. Li, P. Yan, and J. Gu, "Effects of microseismic station location on source location accuracy in microseismic monitoring," in Proceedings of the 3rd International Conference on Civil, Architectural and Hydraulic Engineering (ICCAHE), pp. 638-640, Hangzhou, China, July 2014.

[27] B. X. Jia, L. L. Zhou, Y. S. Pan, and H. Chen, "Artificial seismic source field research on the impact of the number and layout of stations on the microseismic location error of mines," Advances in Civil Engineering, vol. 2019, Article ID 1487486, 11 pages , 2019.

[28] T. A. Stabile, G. Iannaccone, A. Zollo et al., "A comprehensive approach for evaluating network performance in surface and borehole seismic monitoring," Geophysical Journal International, vol. 192, no. 2, pp. 793-806, 2013.

[29] G. D. Landro, M. Picozzi, G. Russo, G. M. Adinolfi, and A. Zollo, "Seismic networks layout optimization for a highresolution monitoring of induced micro-seismicity," Journal of Seismology, vol. 11, pp. 1-14, 2019.

[30] N. Li, M. Ge, and E. Wang, "Two types of multiple solutions for microseismic source location based on arrival-time-difference approach," Natural Hazards, vol. 73, no. 2, pp. 829-847, 2014. 